\title{
Residual strength evaluation under mixed mode loading
}

\author{
Slobodanka Boljanović ${ }^{1,1}$, Stevan Maksimović ${ }^{2}$ and Andrea Carpinteri ${ }^{3}$ \\ ${ }^{1}$ Mathematical Institute of the Serbian Academy of Sciences and Arts, 11000 Belgrade, Serbia \\ ${ }^{2}$ VTI, Aeronautical Department, 11000 Belgrade, Serbia \\ ${ }^{3}$ Department of Engineering and Architecture, University of Parma, 43124 Parma, Italy
}

\begin{abstract}
This work explores the failure resistance of fatigue damages with random orientation through a new computational framework. Such analytical model aims to enable designers to properly and quickly assess stress state and residual life under cyclic loading with constant amplitude and overload, employing suitable fracture mechanics-based concepts. The efficiency of estimates obtained is discussed by means of relevant mixedmode tests available in the literature.
\end{abstract}

\section{Introduction}

Micro-cracks, voids and inclusions, as stress raisers with random orientation, may often increase risk of failure hazard under service loadings. Therefore, the use of computational models for strength performance analysis is indispensable to ensure the structural integrity and full functioning of components.

According to damage tolerance design, random fatigue phenomena, caused by different loading modes, have to be theoretically examined through fracture mechanics criteria: the MTS-criteria [1], the S-criteria [2], the G-criteria [3], the T-criteria [4] and the R-criteria [5, 6]. Furthermore, in order to quantify severity of such modes, relevant crack growth concepts can be taken into account. Tanaka [7] recognized that failure modes should be investigated by means of fracture mechanics parameters and suggested the equivalent stress intensity factor. Sih and Bartelemy [8] introduced the strain energy density concept, whereas Cotterell and Rice [9] employed the energy release rate concept together with the $J$-integral method for analyzing the mixed-mode damages.

Moreover, under service loadings which are usually characterized by variable amplitude, relevant crack growth effects (i.e. retardation, acceleration and reduced retardation due to overload, underload and overload followed by an underload, respectively) have to be analyzed by means of suitable fracture mechanics concepts. Elber [10] introduced the concept of crack opening stress for examining the overload effect and proposed the crack closure model. Wheeler [11] used a fracture mechanics retardation parameter, whereas de Koning et al. [12] developed the strip-yield concept based on the Dugdale's model to evaluate the crack growth delay.

\footnotetext{
${ }^{1}$ Corresponding author: slobodanka.boljanovic@mi.sanu.ac.rs
} 
The purpose of this work is to analyse the fatigue mechanism of mixed mode damages. A new computational framework presented covers the residual life evaluation under constant amplitude loading and overload, and is based on the crack growth law proposed by Zhan et al. combined with the Wheeler's fracture mechanics concept. Furthermore, the influences of relevant mixed mode parameters are taken into account through analytical solutions examined.

\section{Crack growth analysis}

In order to exploit the full potential of cyclically loaded structural components, damages with random orientation should be analyzed through relevant crack growth concepts [13, 14]. The detrimental effects of such crack-like flaws under mixed-mode loading are herein examined by employing the following relationship [15]:

$$
\frac{d a}{d N}=C\left(e^{\alpha R} \Delta K_{e q}\right)^{m}
$$

where $a$ and $N$ are crack length and number of loading cycles, respectively, $R$ is stress ratio, $C$ and $m$ are material parameters experimentally obtained.

In the frame of failure mode assessment the driving forces are taken into account through the equivalent stress intensity factor [7] given by

$$
\Delta K_{e q}=\left(\Delta K_{I}^{2}+2 \Delta K_{I I}^{2}\right)^{0.5}
$$

where $\Delta K_{I}$ and $\Delta K_{I I}$ represent the stress intensity factor range for Mode I and Mode II, respectively.

Then, the fatigue stability is estimated in terms of the number of loading cycles to failure by integrating the relationship for crack growth rate (Eq. 1), as follows:

$$
N=\int_{a_{0}}^{a_{f}} \frac{d a}{C\left(e^{\alpha R} \Delta K_{e q}\right)^{m}}
$$

where $a_{0}$ and $a_{f}$ are initial and final crack length, respectively.

Furthermore, in the mixed mode analysis presented here, the overload phenomenon is also investigated employing the following relationships related to the crack growth rate and number of loading cycles, i.e.

$$
\frac{d a}{d N}=C_{p i} C\left(e^{\alpha R} \Delta K_{e q}\right)^{m}, N=\int_{a_{0}}^{a_{f}} \frac{d a}{C_{p i} C\left(e^{\alpha R} \Delta K_{e q}\right)^{m}}
$$

Note that a delaying of crack growth due to the interaction between the overload plastic zone and the current plastic zone is taken into account through suitable fracture mechanics parameter [11], expressed as follows:

$$
C_{p i}=\left\{\begin{array}{cl}
\left(\frac{r_{p i}}{a_{o l}+r_{p o}-a_{i}}\right)^{p} ; a_{i}+r_{p i} \leq a_{o l}+r_{p o} \\
1 \quad & ; a_{i}+r_{p i} \geq a_{o l}+r_{p o}
\end{array}\right.
$$

where $a_{i}$ and $a_{o l}$ are the current and overload crack length and $p$ is the retardation exponent experimentally obtained. Further, $r_{p i}$ and $r_{p o}$ represent the current plastic zone size and the overload plastic zone size, respectively, which are dependent on the equivalent stress intensity factor range $\Delta K_{e q}$ and the overload stress intensity factor range $\Delta K_{o l}$, as it is discussed in Refs $[13,14]$. 
Under cyclic loading with constant amplitude or overload the fatigue strength is assessed by means of software programmes developed here, in which Euler's numerical integration method is implemented.

\section{Driving force evaluation under mixed mode loading}

The nonlinear behaviour of crack-like flaws under mixed mode loading is theoretically analyzed in terms of the stress intensity factor ranges $\Delta K_{I}$ and $\Delta K_{I I}$ for Mode I and Mode II, respectively, i.e.:

$$
\Delta K_{I}=\frac{\Delta P}{b t} \cos \phi f_{I}\left(\frac{a}{b}\right) \sqrt{\pi a}, \quad \Delta K_{I I}=\frac{\Delta P}{b t} \sin \phi f_{I I}\left(\frac{a}{b}\right) \sqrt{\pi a}
$$

where $\Delta P$ and $t$ are applied force range and thickness, respectively, $a$ is crack length, $b$ and $\phi$ represent the width of specimen and the loading angle.

The boundery conditions related to the geometry of component/specimen with mixedmode damage is taken into account through relevant corrective functions. For the Arcan specimen (Fig. 1a) examined here such relationships can be expressed as follows:

$$
\begin{gathered}
f_{I}\left(\frac{a}{b}\right)=1.12-0.231\left(\frac{a}{b}\right)+10.55\left(\frac{a}{b}\right)^{2}-21.27\left(\frac{a}{b}\right)^{3}+30.39\left(\frac{a}{b}\right)^{4} \\
f_{I I}\left(\frac{a}{b}\right)=\frac{1.122-0.561\left(\frac{a}{b}\right)+0.085\left(\frac{a}{b}\right)^{2}+0.180\left(\frac{a}{b}\right)^{3}}{\left(1-\left(\frac{a}{b}\right)\right)^{0.5}}
\end{gathered}
$$

Moreover, the above corrective function (expressed by Eq. (7)) together with Eq. (6) are also employed to quantify the driving forces for the SENT specimen (Fig. 1b).

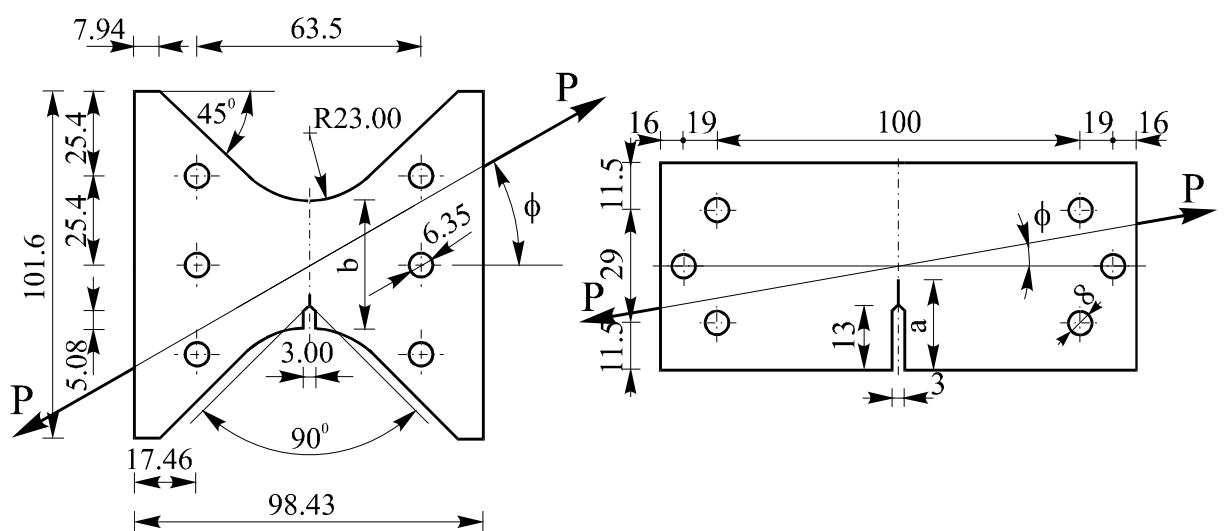

(a)

(b)

Fig. 1. (a) Arcan specimen and (b) SENT specimen. 


\section{Strength assessments of mixed mode damages}

\subsection{Failure performance under cyclic loading with constant amplitude}

Firstly, the behaviour of an Arcan specimen (Fig. 1a) is analyzed under mixed mode loading with constant amplitude. Such residual life evaluations are performed for two applied loading conditions $(R=0.1): \phi=30^{\circ}, P_{\max }=2647 \mathrm{~N}$ and $\phi=75^{\circ}, P_{\max }=3047 \mathrm{~N}$. The specimen (characterized by the following sizes: $t=1.6 \mathrm{~mm}, b=38.1 \mathrm{~mm}, a_{0}=2.997$ $\mathrm{mm})[14,16]$ is made of $2024 \mathrm{~T} 351$ aluminium alloy $\left(C=5.310^{-10}, m=2.9\right)$.

Under mixed mode loading conditions, fatigue strength is herein estimated by employing Eqs (2) and (3) together with Eqs (6) - (8). The evaluated residual life, as a function of crack length, is shown in Fig. $2 \mathrm{a}$ and $\mathrm{b}$ for applied loadings characterized by $\phi=30^{\circ}, P_{\max }=2647 \mathrm{~N}$ and $\phi=75^{\circ}, P_{\max }=3047 \mathrm{~N}$, respectively. Further, in Table 1 such mixed mode results are compared with experimental observations for two material orientations (that is longitudinal transverse (LT) and transverse longitudinal (TL)) [16]. Relevant comparisons indicate that in the case of loading angle equal to $30^{\circ}$ the conservative trend of estimates (with respect to experiments) is obtained for both material orientations, whereas if the loading angle is equal to $75^{\circ}$ the conservative result is evaluated in the case of the LT material orientation. Further, from Fig. 2 and Table 1 it can be seen that mixed mode effects under loading angle equal to $30^{\circ}$ threaten less the life to failure than those in the case of loading angle equal to $75^{\circ}$.
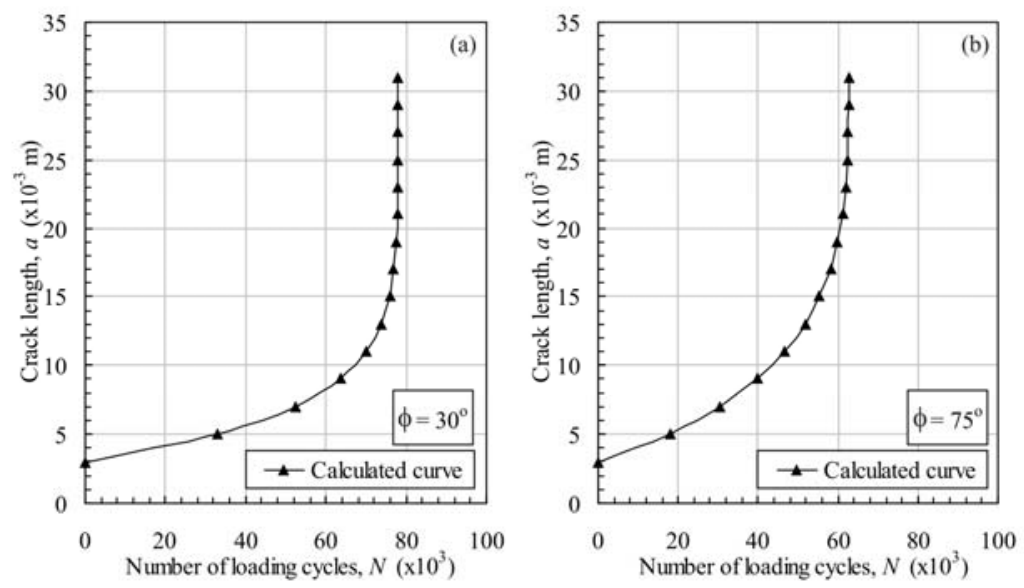

Fig. 2. Residual life analysis: (a) $\phi=30^{\circ}, P_{\max }=2647 \mathrm{~N}$ and (b) $\phi=75^{\circ}, P_{\max }=3047 \mathrm{~N}$ (calculated curve - the present results).

Table 1 Evaluated and experimental number of loading cycles [16].

\begin{tabular}{|c|c|c|}
\hline & \multicolumn{2}{|c|}{$N($ cycles) } \\
\hline$\phi\left(^{\circ}\right)$ & Evaluated & Experiment [16] \\
\hline 30 & 77990 & 87131 (IISA40TL), 120875(IISA59LT), 169670 (IISA66TL), \\
\hline 75 & 62770 & 57500 (IISA38TL), 96328 (IISA60TL), 110000 (IISA27LT) \\
\hline
\end{tabular}




\subsection{Failure performance under overload}

Now the mixed mode failure under overload is theoretically examined. In such fatigue analysis, for the SENT specimen (Fig. 1b) made of 2024 T3 aluminium alloy, the following geometrical and material parameters are assumed: $a_{0}=17.75 \mathrm{~mm}, b=52 \mathrm{~mm}, t=6.5 \mathrm{~mm}$ $[13,14], \sigma_{y s}=324 \mathrm{MPa}, E=73100 \mathrm{MPa}, C=1.61 \times 10^{-10}, m=2.94$. Note that, calculations herein presented are carried out in the case of two different loading angles $\left(\phi=18^{\circ}\right.$ and $\left.54^{\circ}\right)$ with maximum force equal to $P_{\max }=7197 \mathrm{~N}(R=0.1)$ associated with overload $\left(a_{o l}=20.40 \mathrm{~mm}, P_{\text {maxol }}=17993 \mathrm{~N}, R_{o l}=2.5\right)$.

In order to assess the fatigue stability under overload the fracture mechanics-based analytical model is taken into account, as is discussed in previous sections. Relevant equivalent stress intensity factors are evaluated through Eq. (2) together with Eqs (6) and (7). Then, the failure strength is estimated in terms of the number of loading cycles by employing Eqs (4) and (5). Such mixed-mode results for loading angle $\phi=18^{\circ}$ and $54^{\circ}$ (together with those found in the literature [13]) are shown in Fig. 3a and b, respectively. It can be seen that results related to fatigue life are in a quite good agreement.
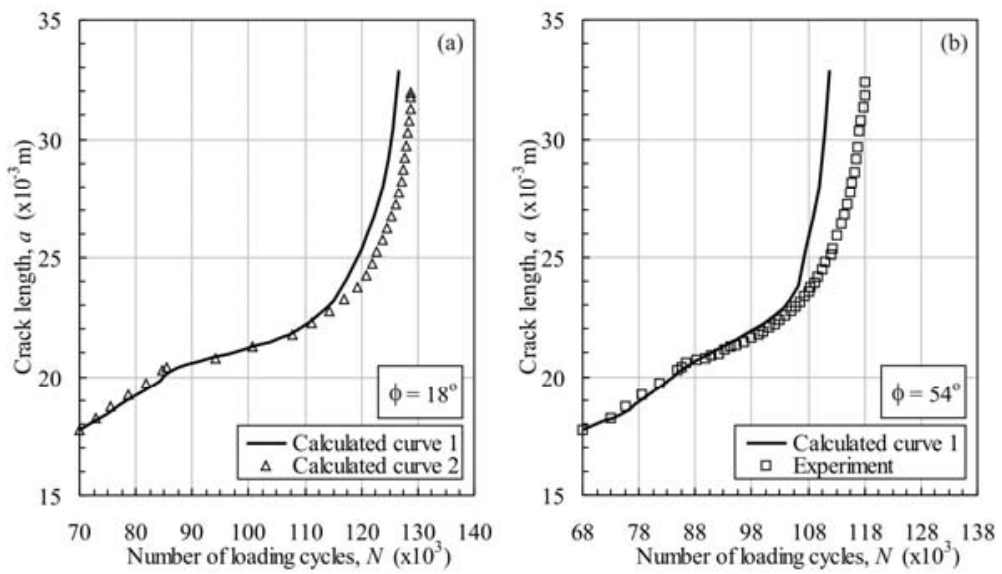

Fig. 3. Residual life analysis under overload ( $P_{\text {maxol }}=17993 \mathrm{~N}$ ): (a) $\phi=18^{\circ}$ and (b) $\phi=54^{\circ}$ (calculated curve 1 - the present results, calculated curve 2 and experiment are reported in Ref. [13]).

\subsection{The effects of stress ratio, overload stress ratio, thickness and width}

Finally, mixed-mode evaluations examine the effect of overload stress ratio and thickness on the fatigue resistance of the SENT specimen (Fig. 1b), made of 2024 T3 aluminium alloy. In such analysis three specimens $\left(a_{0}=19 \mathrm{~mm}, b=55 \mathrm{~mm}, t=7 \mathrm{~mm}\right)$ are subjected to cyclic loading $\left(\phi=18^{\circ}, P_{\max }=6000 \mathrm{~N}, R=0.1\right)$ together with three different overloads $\left(a_{o l}=21.50 \mathrm{~mm}, P_{\text {maxol }}=16000 \mathrm{~N}\right)$ characterized by three different stress ratios: $R_{o l}=2.5$, 3.75 and 5, respectively. Further, the failure behaviour of the same damaged specimen ( $a_{0}=19 \mathrm{~mm}, b=55 \mathrm{~mm}, t=6 \mathrm{~mm}$ ) is investigated by assuming three different stress ratios $(R=0.1 \mathrm{~mm}, 0.25$ and 0.5$)$, whereas applied loading is characterized by the following parameters: $\phi=54^{\circ}, P_{\max }=7000 \mathrm{~N}, a_{o l}=21.50 \mathrm{~mm}, P_{\text {maxol }}=16000 \mathrm{~N}, R_{o l}=2.5$.

Moreover, for the Arcan specimen (Fig. 1a) subjected to mixed mode loading with constant amplitude, the fatigue stability is assessed by taking into account the effect of thickness and width. Actually, the residual strength analysis is performed for two specimen configurations $\left(a_{0}=4.347 \mathrm{~mm}, b=38.1 \mathrm{~mm}, t=2 \mathrm{~mm}\right)$ with three different thicknesses $(t=1.5 \mathrm{~mm}, 1.8 \mathrm{~mm}, 2.16 \mathrm{~mm})$ and with three widths $(b=36 \mathrm{~mm}, 41.4 \mathrm{~mm}, 47.61 \mathrm{~mm})$ by 
adopting the following applied loadings $(R=0.1): \phi=30^{\circ}, P_{\max }=2800 \mathrm{~N}$ and $\phi=45^{\circ}$, $P_{\max }=3000 \mathrm{~N}$, respectively. Note that, for materials examined here the same fatigue parameters are assumed as those mentioned in above sections.

The evaluated numbers of loading cycles versus crack length in the case of overload and for cyclic loading with constant amplitude are shown in Fig. 4 and 5, respectively. By examining such figures it can be inferred that stress ratio, overload stress ratio, thickness and width significantly affect the residual strength under mixed mode loading with/without overload.
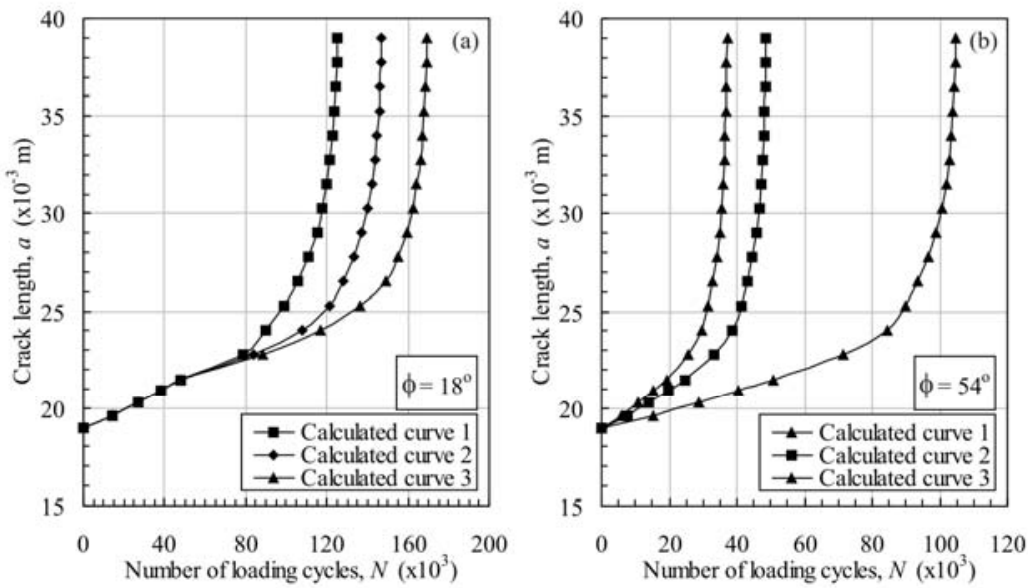

Fig. 4. Residual life analysis: (a) $1-R_{o l}=2.5,2-R_{o l}=3.75,3-R_{o l}=5$ and (b) $1-R=0.1,2-$ $R=0.25,3-R=0.5$.
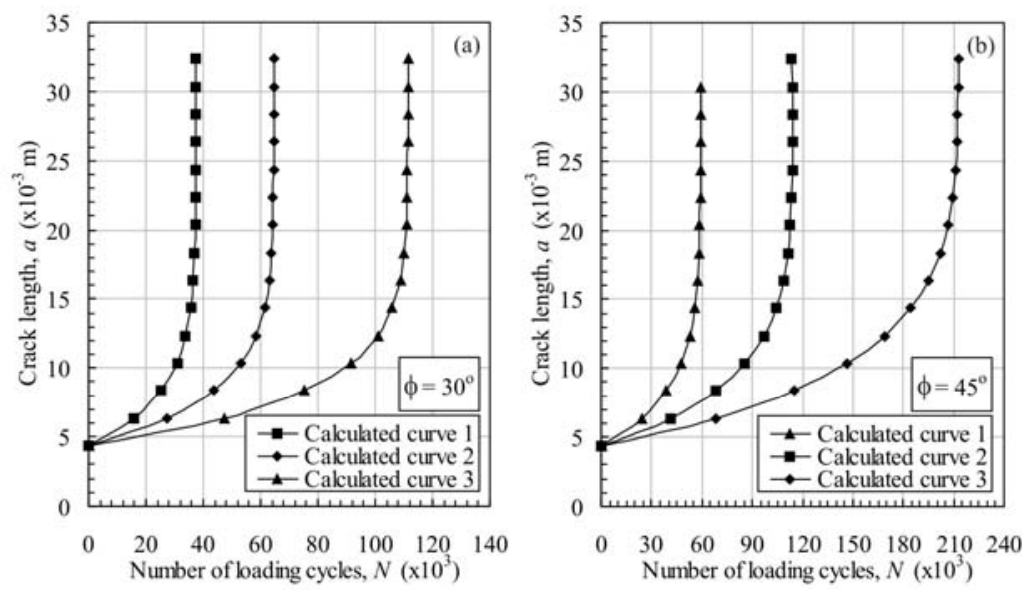

Fig. 5. Residual life analysis: (a) $1-t=1.5 \mathrm{~mm}, 2-t=1.8 \mathrm{~mm}, 3-t=2.16 \mathrm{~mm}$ and (b) $1-w=$ $36 \mathrm{~mm}, 2-w=41.4 \mathrm{~mm}, 3-w=47.61 \mathrm{~mm}$.

\section{Conclusions}

The ability to assess the failure resistance of fatigue damages with random orientation is an important issue in automotive and aerospace industries. Therefore, this paper describes a new fracture mechanics-based analytical model for evaluating the stress state and residual life under mixed mode loading, in which extended relationship proposed by Zhan et al. 
together with Wheeler's crack growth concept are implemented. One of the major benefits of such framework in exploring random fatigue mechanisms is that it can be employed in the case of constant amplitude loading and/or overload.

The research presented was financially supported by the Mathematical Institute of the Serbian Academy of Sciences and Arts and the Ministry of Science and Technological Development of Serbia through the project No. OI 174001.

\section{References}

1. F. Erdogan, G.C. Sih, On the crack extension in plates under plane loading and transverse shear, J Basic Eng 85: 519-27 (1963).

2. G.C. Sih, Strain-energy-density factor applied to mixed mode crack problems, Int J Fract 10: 305-21 (1974).

3. M.A. Hussain, S.L. Pu, J. Underwood, Strain-energy-release rate for a crack under combined mode I and mode II, ASTM STP vol.560, Am Soc Test Mater, Philadelphia, PA, p.2-28 (1974).

4. P.S. Theocaris, N.P. Andrianopoulos, The T-criterion applied to ductile fracture, Int J Fract 20:R125-30 (1982).

5. M.A.K. Shafique, K.K. Marwan, Analysis of mixed mode crack initiation angles under various loading conditions, Eng Fract Mech 67:397-419 (2000).

6. A. Carpinteri, A. Spagnoli, S. Vantadori, A multiaxial criterion for notch high-cycle fatigue using a critical-point method, Eng Fract Mech 75(7) :1864-74 (2008).

7. K. Tanaka, Fatigue crack propagation from a crack inclined to the cyclic tensile axis, Eng Fract Mech 6:493-507 (1974).

8. G.C. Sih, B.M. Barthelemy, Mixed mode fatigue crack growth predictions, Eng Fract Mech 13:439-51 (1980).

9. B. Cotterell, J.R. Rice, Slightly curved or kinked cracks, Int J Fract 16:155-69 (1980).

10. W. Elber, Fatigue crack closure under cyclic tension, Eng Fract Mech 2:37-45 (1970).

11. O.E. Wheeler, Spectrum loading and crack growth, J Basic Eng 94: 181-6 (1972).

12. A.U. de Koning, H.J. ten Hoeve, T.K. Hendriksen, The description of crack growth on the basis of the strip-yield model for computation of crack opening loads, the crack tip stretch and strain rates, National Aerospace Lab., Report NLR-TP-97511L (1997).

13. J.R. Mohanty, B.B. Verma, P.K. Ray, Prediction of fatigue life with interspersed mode-I and mixed-mode (I and II) overloads by an exponential model: extensions and improvements, Eng Fract Mech 76 :454-68 (2009).

14. S. Boljanović, S. Maksimović, Computational mixed mode failure analysis under fatigue loadings with constant amplitude and overload, Eng Fract Mech 174:168-179 (2017).

15. W. Zhan, N. Lu, C. Zhang, A new approximate model for the R-ratio effect on fatigue crack growth rate, Eng Fract Mech 119:85-96 (2014).

16. S.E. Galyon, S.R. Arunachalam, J. Greer, M. Hammond, S.A. Fawaz, Three dimensional crack growth prediction, In: M. Bos, editor: Bridging the gap between theory and operational practice, New York, Springer, ICAF, p.1035-68 (2009). 